\title{
Luminosity-Colour Relations for Red Clump Stars
}

\author{
S. Bilir ${ }^{1}$ • Ö. Önal ${ }^{1} \bullet$ S. Karaali ${ }^{1}$ • \\ A. Cabrera-Lavers ${ }^{2,3} \cdot$ H. Çakmak ${ }^{1}$
}

(C) Springer-Verlag $\bullet \bullet \bullet \bullet$

\begin{abstract}
We calibrated the $M_{V}, M_{J}, M_{K_{s}}$ and $M_{g}$ absolute magnitudes of red clump stars in terms of colours. $M_{V}$ and $M_{g}$ are strongly dependent on colour, while the dependence of $M_{J}$ and $M_{K_{s}}$ on colour is rather weak. The calibration of $M_{V}$ and $M_{K_{s}}$ absolute magnitudes is tested on $101 \mathrm{RC}$ stars in the field SA 141. The Galactic model parameters estimated with this sample are in good agreement with earlier studies.
\end{abstract}

Keywords Stars: distances, Stars: late-type, Galaxy: fundamental parameters

\section{Introduction}

Red Clump (RC) stars are core helium-burning giants. They form a prominent feature in the colourmagnitude diagrams (CMDs) of open clusters. Fol-

\section{S. Bilir}

${ }^{1}$ Istanbul University, Faculty of Science, Department of Astronomy and Space Sciences, 34119 University, Istanbul, Turkey

Ö. Önal

${ }^{1}$ Istanbul University, Faculty of Science, Department of Astronomy and Space Sciences, 34119 University, Istanbul, Turkey

S. Karaali

${ }^{1}$ Istanbul University, Faculty of Science, Department of Astronomy and Space Sciences, 34119 University, Istanbul, Turkey

\footnotetext{
A. Cabrera-Lavers

${ }^{2}$ Instituto de Astrofísica de Canarias, E-38205 La Laguna, Tenerife, Spain

${ }^{3}$ Departamento de Astrofísica, Universidad de La Laguna, E38205 La Laguna, Tenerife, Spain

H. Çakmak

${ }^{1}$ Istanbul University, Faculty of Science, Department of Astronomy and Space Sciences, 34119 University, Istanbul, Turkey
}

lowing the prediction of Cannon (1970), it is known that they are abundant in the solar neighbourhood. In recent years much work has been devoted to studying the suitability of RC stars as a distance indicator. Their absolute magnitudes in the optical range lie from $M_{V}=+0.7$ mag for those of spectral type G8 III to $M_{V}=+1 \mathrm{mag}$ for type K2 III (Keenan \& Barnbaum 1999). The absolute magnitude of these stars in the $K_{s}$ band is $M_{K_{s}}=-1.61 \pm 0.03 \mathrm{mag}$ with negligible dependence on metallicity (Alves 2000), but with real dispersion. Based on observations of 14 open clusters with $-0.5<[\mathrm{Fe} / \mathrm{H}] \leq 0$ dex and $1.58 \leq t \leq 7.94 \mathrm{Gyr}$, Grocholski \& Sarajedini (2002) found that for RC stars in clusters have $\left\langle M_{K_{s}}\right\rangle=-1.61 \pm 0.04 \mathrm{mag}$.

The dependence of the $I$-band magnitude of RC stars on the metallicity and age was extensively studied in the past from an observational point of view. In the most cases the $I$ band-mean absolute magnitude is insensitive to age and metallicity. Udalski (2000) found that the $M_{I}$ of RC stars weakly depends on metallicity. Paczvnski \& Stanek (1998) and Stanek \& Garnavich (1998) found little or no variation in $M_{I}$ with colour and metallicity. Sarajedini (1999) presented observations of eight open clusters, concluding that $M_{I}$ is less sensitive to metal abundance than $M_{V}$, but that the dependence on age and metallicity is still not negligible. Zhao et al. (2001) and Kubiak et al. (2002) confirmed the results of Udalski (2000), and theoretical models from Girardi \& Salaris (2001) also show a dependence in $I$-band, predicting that an older cluster with higher metallicity has fainter RC stars. Based on the model of Girardi et al. (2000), Salaris \& Girardi (2002) stated that $M_{K}$ is a complicated function of metallicity and age. For age $t>1.5 \mathrm{Gyr}$, it decreases with increasing metallicity, the opposite behaviour with respect to $M_{V}$ and $M_{I}$ absolute magnitudes.

Pietrzynski et al. (2003) have also investigated the dependence of the mean $K, J$, and $I$ absolute magni- 
tudes of the RC stars on metallicity and age, as a part of their ongoing Araucaria Project to improve stellar distance indicators. They took deep near-infrared (NIR) $J$ and $K$ images of several fields in LMC, SMC, and the Carina, and Fornax dwarf galaxies and made a comparison between the extinction -corrected $K$-band RC star magnitudes and some other stellar indicators, particularly the tip of the red giant -branch magnitude, the mean RR Lyrae star $V$-band magnitude, and the mean $K$-band magnitude of Cepheid variables at a period of 10 days. This comparison strongly suggests that absolute $K$-band magnitude of the RC stars have a very weak dependence, (if any) on $[\mathrm{Fe} / \mathrm{H}]$ over the broad range of metallicities covered by their target galaxies. They conclude that the mean $K$-band magnitude of the $\mathrm{RC}$ stars is an excellent distance indicator with small (if any) population corrections over a wide range in metallicity and age. Pietrzynski et al. (2010) stated that population effects strongly affect both $V$-and $I$ - band magnitude of RC stars in a complicated way. Therefore, optical $V-I$ photometry of RC stars is not an accurate method for the determination of distances in nearby galaxies, while NIR photometry is a much better way to measure distances with $\mathrm{RC}$ stars given its smaller sensitivity to population effects. Laney et al. (2012) determined the mean $M_{K_{s}}$ absolute magnitude for RC stars in the solar neighbourhood to within 2 per cent $\left(M_{K_{s}}=-1.613 \pm 0.015 \mathrm{mag}\right)$ and applied their results to the estimation of the distance of LMC. A mean value for the $M_{K_{s}}$ absolute magnitude with weak dependence on metallicity makes it possible to use this population as a tracer of Galactic structure and interstellar extinction, as several works have fully demonstrated in the last decade (see for example Lopez-Corredoira et al. 2002, 2004; Cabrera-Lavers et al. 2005, 2007a, b, 2008; Bilir et al. 2012, and references therein).

In a recent work, van Helshoecht \& Groenewegen (2007) used the Two Micron All Sky Survey (2MASS; Skrutskie et al. 2006) infrared data for a sample of 24 open clusters to investigate how the $K_{s}$-band absolute magnitude of the red clump depends on age and metallicity. They showed that a constant value of $M_{K_{s}}=-1.57 \pm 0.05 \mathrm{mag}$ is a reasonable assumption to use in distance determinations of clusters with metallicity between -0.5 and +0.4 dex and age between 0.31 and 7.94 Gyr. The constant absolute magnitude value of $\mathrm{RC}$ stars was also supported with the newly reduced Hipparcos data by Groenewegen (2008) ( $\left.M_{K_{s}}=-1.54 \pm 0.04 \mathrm{mag}\right)$.

In this paper, we will contribute to the discussion by a different approach. We aim to calibrate the absolute magnitudes of RC stars in four bands, i.e. $V$, $J, K_{s}$ and $g$, as a function of colour with field stars taken from different photometric surveys. The data are given in Section 2. The location of the RC stars in the Hertzsprung-Russell (H-R) diagram are demonstrated in Section 3. The luminosity-colour relations in optical and near-infrared are given in Sections 4. We tested the absolute magnitudes derived by the procedure in our study in Section 5, and a summary and conclusion is presented in Section 6.

\section{The data}

We used three sets of data. The BVI data were taken from the Hipparcos catalogue van Leeuwen 2007) which also provides trigonometric parallaxes. In order to obtain reliable absolute magnitude and distances, only the stars with relative parallax error $\left(\sigma_{\pi} / \pi\right)$ is less than or equals to 0.1 are selected. Thus, 32144 stars are included into the sample with $B V I$ magnitudes. The second set of data consists of $J H K_{s}$ magnitudes. Within this sample only 32072 stars were detected in the 2MASS catalogue (Cutri et al. 2003). The Hipparcos stars were not observed in the Sloan Digital Sky Survey (SDSS; York et al. 2000). Hence, the gri magnitudes of the sample stars were evaluated by the transformation equations of Yaz et al. (2010). Thus, the resulting catalogue consisted of three different photometries, i.e. Johnson-Cousins $(B V I)$, 2MASS $\left(J H K_{s}\right)$, and SDSS (gri) of all the RC stars within the Solar neighbourhood.

\subsection{Lutz-Kelker Correction}

The observed trigonometric parallaxes are biased because the volume of space per unit of parallax is not constant. The pioneers of this topic are Trumpler \& Weaver (1953). However, Lutz \& Kelker (1973) were the first who quantified the bias. Other studies followed the work of Lutz \& Kelker (1973). Smith (1987) claimed that Lutz \& Kelker (1973)'s methodology seemed to be that of Bayesian statistics. The Luzt-Kelker (LK) bias can be explained as follows. Let $\pi$ and $\sigma_{\pi}$ be the parallax and its error of a star. Then, one can define a distance $d$ with lower and upper limits, i.e. $d_{\pi+\sigma_{\pi}}$ and $d_{\pi-\sigma_{\pi}}$. Stars in a given volume can scatter to the distance $d$. Since the number of stars in the distance interval $\left(d, d_{\pi-\sigma_{\pi}}\right]$ are more than the ones in the distance interval $\left[d_{\pi+\sigma_{\pi}}, d\right]$, more stars from the interval $\left(d, d_{\pi-\sigma_{\pi}}\right]$ will scatter to distance $d$ than the ones in $\left[d_{\pi+\sigma_{\pi}}, d\right]$. The result of this effect is that measured parallaxes cause smaller distance estimations. We used 
the following equation of Smith (1987) to correct the observed Hipparcos parallaxes (van Leeuwen 2007),

$\pi_{0}=\pi\left(\frac{1}{2}+\frac{1}{2} \sqrt{1-16\left(\sigma_{\pi} / \pi\right)^{2}}\right)$,

where $\pi$ and $\pi_{0}$ are the observed and corrected parallaxes, respectively, and $\sigma_{\pi}$ denotes the error of the observed parallax. According to Lutz \& Kelker (1973) relative parallax errors, $\sigma_{\pi} / \pi$, larger than 0.17 are not reliable.

\subsection{Reddening}

The $E(B-V)$ colour excess of stars have been evaluated in two steps. First, we used the maps of Schlegel et al. (1998) and evaluated a $E_{\infty}(B-V)$ colour excess for each star. Then, we reduced them using the following procedure (Bahcall \& Soneira 1980):

$A_{d}(b)=A_{\infty}(b)\left[1-\exp \left(\frac{-|d \times \sin (b)|}{H}\right)\right]$.

Here, $b$ and $d$ are the Galactic latitude and distance to the star, respectively. $H$ is the scale height for the interstellar dust which is adopted as $125 \mathrm{pc}$ (Marshall et al. 2006). $A_{\infty}(b)$ and $A_{d}(b)$ are the total absorptions for the model and for the distance to the star, respectively. $A_{\infty}(b)$ can be evaluated by means of the following equation:

$A_{\infty}(b)=3.1 \times E_{\infty}(B-V)$.

$E_{\infty}(B-V)$ is the colour excess for the model taken from the Schlegel et al. (1998). Then, $E_{d}(B-V)$, i.e. the colour excess for the corresponding star at the distance $d$, can be evaluated via equation,

$E_{d}(B-V)=A_{d}(b) / 3.1$.

We have omitted the indices $\infty$ and $d$ from the colour excess $E(B-V)$ in the equations. However, we use the terms "model" for the colour excess of Schlegel et al. (1998) and "reduced" for the colour excess corresponding to distance $d$. The total absorption $A_{d}$ used in the section and classical total absorption $A_{V}$ have the same meaning.

We de-reddened the colours and magnitudes using the $E(B-V)$ colour excesses of the stars evaluated from the procedures explained above and the following equations from Fan (1999) and Fiorucci \& Munari (2003) for $V-I$ colour and for the 2MASS photometry.

$$
\begin{array}{r}
V_{o}=V-3.1 \times E(B-V), \\
(B-V)_{o}=(B-V)-E(B-V), \\
(V-I)_{o}=(V-I)-1.250 \times E(B-V), \\
J_{o}=J-0.887 \times E(B-V), \\
(J-H)_{o}=(J-H)-0.322 \times E(B-V), \\
\left(H-K_{s}\right)_{o}=\left(H-K_{s}\right)-0.183 \times E(B-V) .
\end{array}
$$

\section{Hertzsprung-Russell Diagram of RC Stars}

We evaluated the $V$-band absolute magnitudes of 32 144 Hipparcos stars with relative parallax errors less than 0.1 using the following formula and plotted them onto the H-R diagram (Fig. 1) to identify the location of the RC stars:

$M_{V}=V_{0}-5 \log \frac{1000}{\pi(\text { mas })}-5$.

The sample stars were fitted to Padova isochrones (Marigo et al. 2008) with metallicities $[M / H]=0$, -0.5 , and -1 dex and ages $t=1,5$, and 10 Gyr. The relatively high condensed region on the evolved segments of the isochrones corresponds to the location of the RC. The borders of the RC stars in the vertical direction have been fixed by using the constraint of Puzeras et al. (2010), i.e. $2.1 \leq \log g \leq 2.7$, where $g$ denotes surface gravity of the RC stars. These borders comprise the region in which $\mathrm{RC}$ stars identified in the literature. However, for the borders in the horizontal direction we used their highly concentrated location, i.e. $0.7 \leq(B-V)_{0} \leq 1.3$. The number of the RC stars defined in this way are 2576 . We separated them into three categories with respect to their Galactic latitudes, i.e. $|b| \leq 30^{\circ}, 30^{\circ}<|b| \leq 60^{\circ}$, and $|b|>60^{\circ}$. Their median $E(B-V)$ colour excesses are $0.035,0.020$, and $0.012 \mathrm{mag}$, respectively (Fig. 2). These stars will be used in the following sections to derive colour dependent $M_{V}$ absolute magnitude calibrations.

The distances of $2576 \mathrm{RC}$ stars evaluated by the combination of their absolute and de-reddened apparent $V_{0}$ magnitudes show almost a symmetrical distribution (Fig. 3). The median distance is 140 pc. Distribution of stars in $X-Y$ and $X-Z$ planes is given in Fig. 4, where $X, Y$, and $Z$ are the heliocentric rectangular coordinates. The distribution of stars in the medians of $X, Y$, and $Z$, i.e. $+3,-1$, and -5 pc, respectively show that the distribution of RC stars in the solar neighbourhood is almost symmetrical.

The errors of magnitudes in 2MASS photometry are larger than the optical ones, $B, V$, and $I$. We selected 


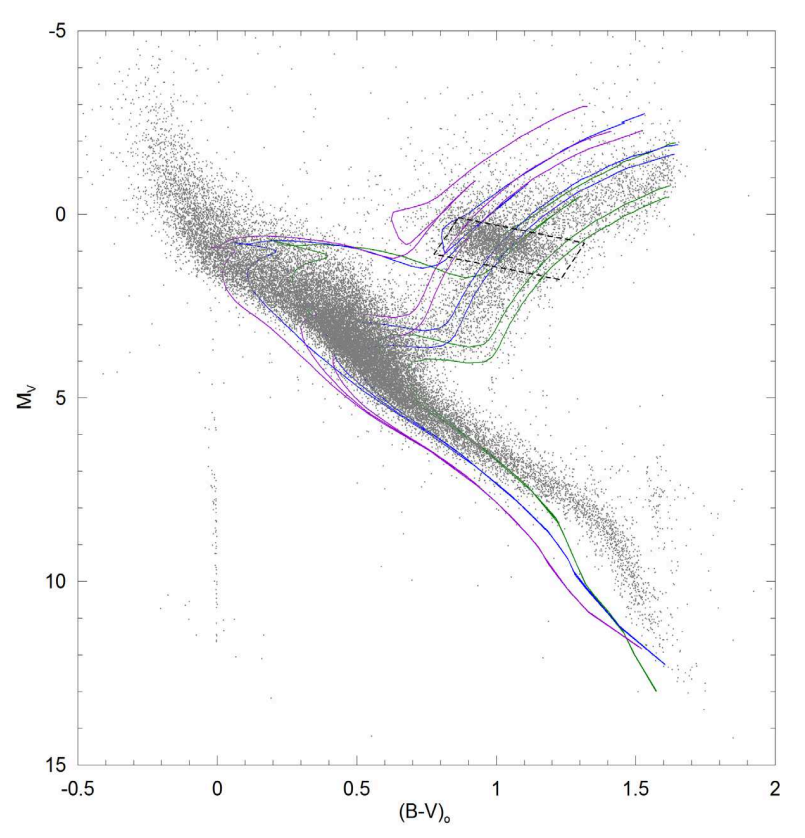

Fig. 1 The $M_{V}-(B-V)_{0}$ diagram for 32144 stars, taken from the Hipparcos catalogue, fitted to the Padova isochrones with metallicities $[M / H]=0,-0.5$, and -1 dex, and ages $t=1,5$, and $10 \mathrm{Gyr}$. The dotted lines show the location of RC stars.

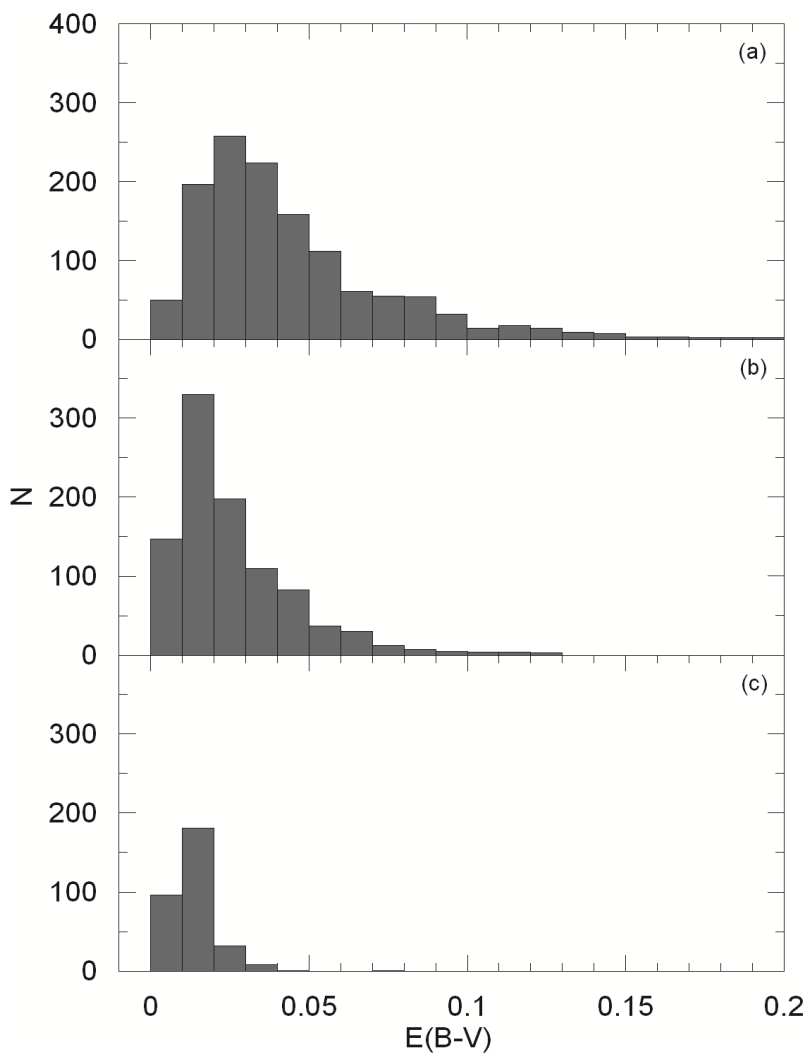

Fig. 2 Distribution of the $E(B-V)$ colour excesses of $2576 \mathrm{RC}$ stars for three Galactic latitudes, $|b| \leq 30^{\circ}$ (a), $30^{\circ}<|b| \leq 60^{\circ}(\mathrm{b})$, and $|b|>60^{\circ}$ (c).

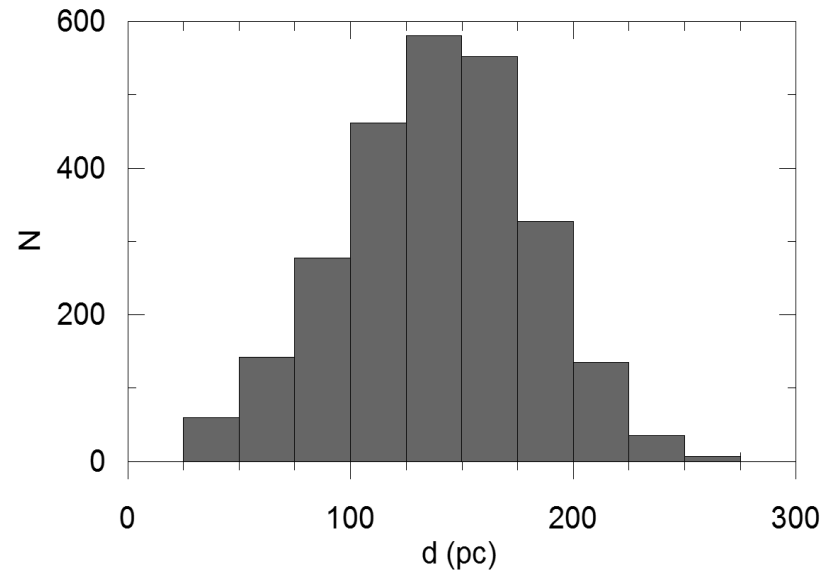

Fig. 3 Distance distribution of 2576 RC stars. The median of the distances is $d=140 \mathrm{pc}$.

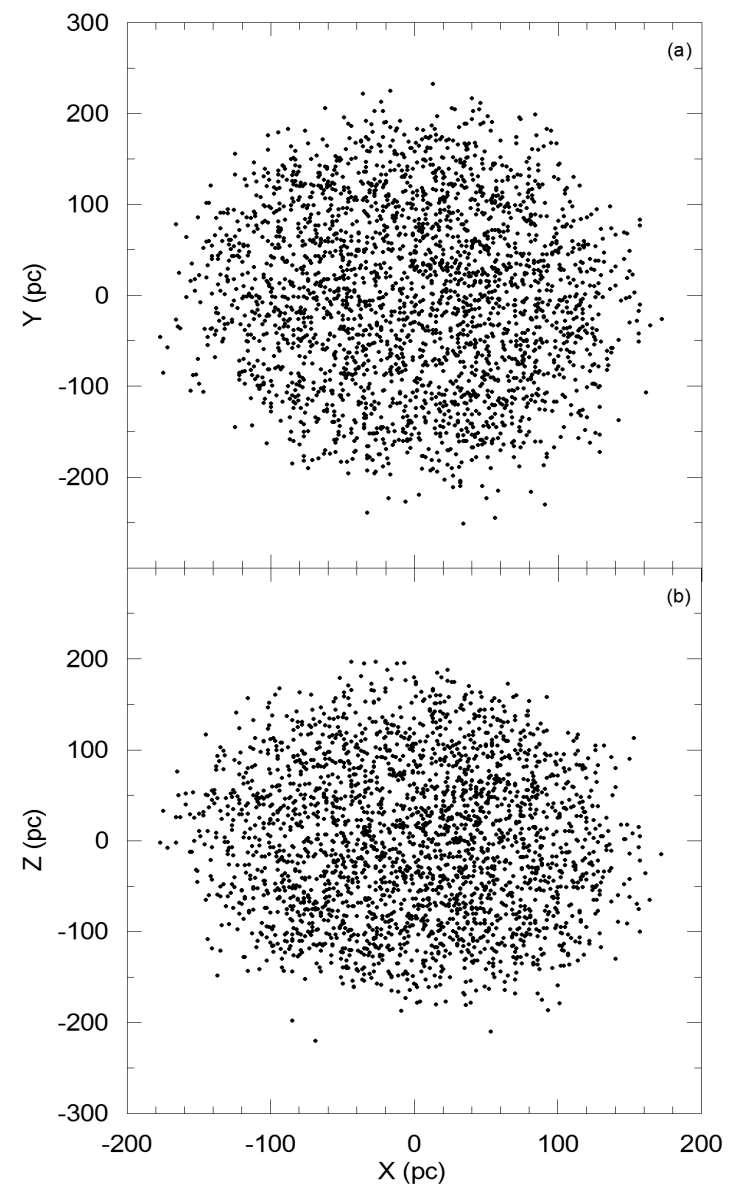

Fig. 4 Distributions of $2576 \mathrm{RC}$ stars in the $X-Y$ and $X-Z$ planes. 
the best quality $J, H$, and $K_{s}$ magnitudes by applying the constraint "AAA" to avoid the large errors. We found 25401 stars using this criteria. Then, we evaluated the $M_{J}$ and $M_{K_{s}}$ absolute magnitudes by means of the procedure applied to $M_{V}$ absolute magnitudes, and plotted them onto the H-R diagram, $M_{J}-(J-H)_{o}$ and $M_{K_{s}}-\left(J-K_{s}\right)_{o}$, respectively, to identify the location of the RC stars (Fig. 5 and Fig. 6) with $J$ and $K_{s}$ bands. As in $M_{V}$, the sample stars were fitted to the Padova isochrones (Marigo et al. 2008) mentioned above. The number of RC stars in Fig. 5 and Fig. 6 $(N=499)$ are less than the ones in Fig. $1(N=2576)$, due to the constraint "AAA" used to ensure the best photometric quality.

The errors of the photometric data are given in Fig. 7. One can see that the errors for the optical colour and magnitudes are smaller than the corresponding NIR ones. Also, the errors for bright magnitudes are lower than the faint ones, as expected, and the errors for $(B-V)_{o}$ colours are rather smaller than the ones for $(V-I)_{o}$ colours.

We do not plot any H-R diagram for the SDSS (gri) data, because they were provided by the transformation equations, as mentioned above. The equation used to evaluate the $M_{a}$ absolute magnitude is given in the following (Yaz et al. 2010):

$M_{g}-M_{J}=2.923(J-H)_{0}+3.031\left(H-K_{s}\right)_{0}+0.329$.

\section{Luminosity-Colour Relations for RC Stars}

We evaluated two different sets of colours for each star sample mentioned above, i.e. $(B-V)_{o}$ and $(V-I)_{o}$; $(J-H)_{o}$ and $\left(H-K_{s}\right)_{o} ;(g-r)_{o}$ and $(r-i)_{o}$ for JohnsonCousins, 2MASS and SDSS photometries, respectively, and combined them with the corresponding absolute magnitudes $\left(M_{V}, M_{J}, M_{K_{s}}\right.$, and $\left.M_{g}\right)$ evaluated by means of their apparent magnitudes and Hipparcos parallaxes. We separated the $(B-V)_{o}$ and $(V-I)_{o}$ colours into 11 bins, whereas only six bins could be provided for the $(J-H)_{o},\left(H-K_{s}\right)_{o},(g-r)_{o}$ and $(r-i)_{o}$ colours due to smaller number of stars in 2MASS and SDSS photometries. Table 1 gives the mean colours and absolute magnitudes of the bins in question. Then, we adapted the colours and absolute magnitudes in Table 1 to the following equations and obtained colour dependent absolute magnitude equations for the RC stars by regression analysis.

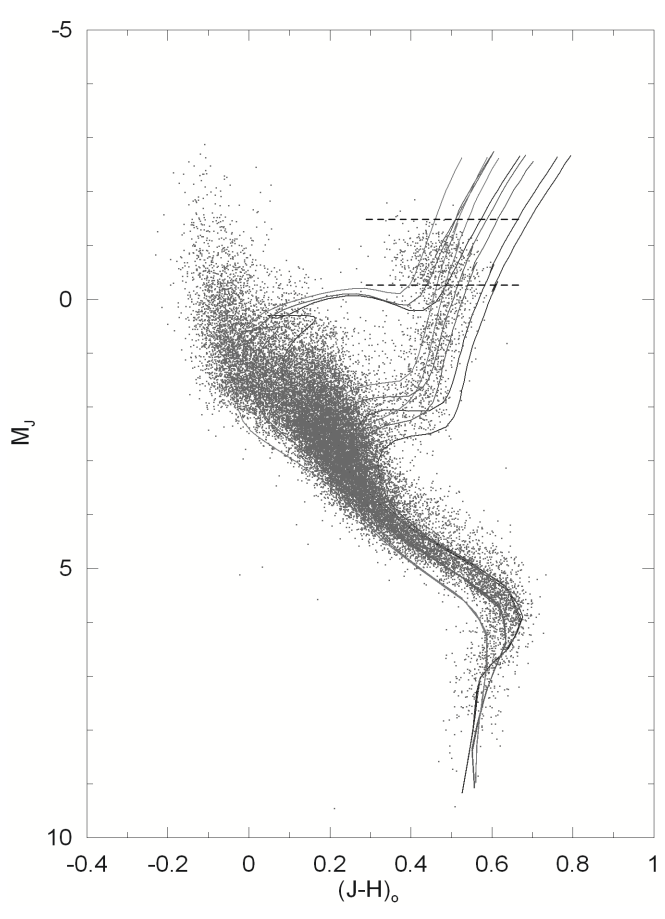

Fig. 5 The $M_{J}-(J-H)_{0}$ diagram for 25104 stars, taken from the Hipparcos catalogue, fitted to the Padova isochrones with metallicities $[M / H]=0,-0.5$, and $-1 \mathrm{dex}$, and ages $t=1,5$, and 10 Gyr. The dotted lines show the location of the RC stars.

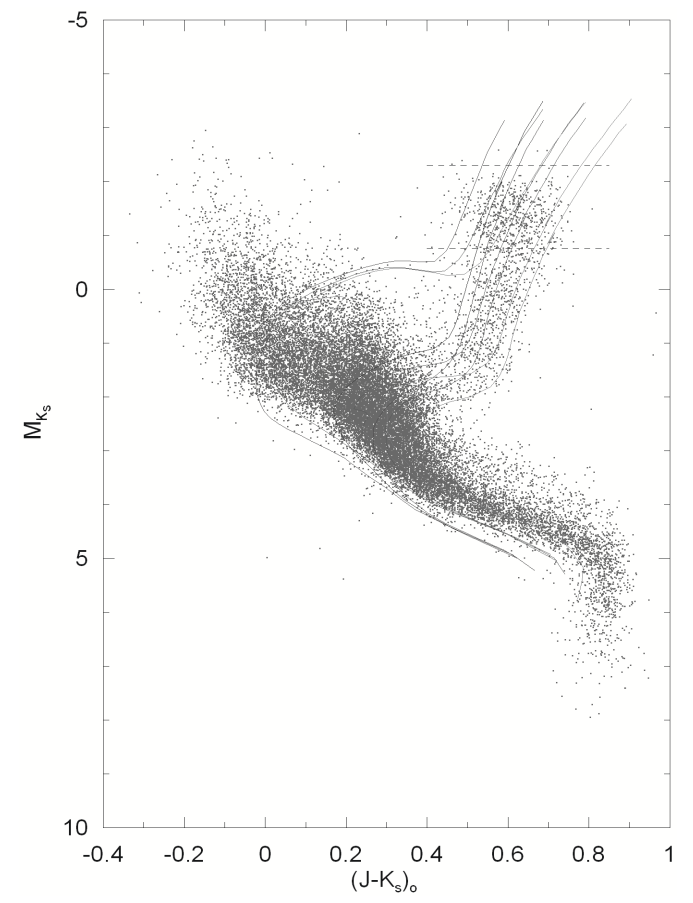

Fig. 6 The $M_{K_{s}}-\left(J-K_{s}\right)_{o}$ diagram for 25104 stars, taken from the Hipparcos catalogue, fitted to the Padova isochrones with metallicities $[M / H]=0,-0.5$, and -1 dex, and ages $t=1,5$, and 10 Gyr. The dotted lines show the location of the RC stars. 


$$
\begin{array}{r}
M_{V}=a_{1}(B-V)_{0}+b_{1}(V-I)_{0}+c_{1}, \\
M_{g}=a_{2}(g-r)_{0}+b_{2}(r-i)_{0}+c_{2}, \\
M_{J}=a_{3}(J-H)_{0}+b_{3}\left(H-K_{s}\right)_{0}+c_{3}, \\
M_{K_{s}}=a_{4}(J-H)_{0}+b_{4}\left(H-K_{s}\right)_{0}+c_{4} .
\end{array}
$$

The numerical values of the coefficients $a_{i}, b_{i}$, and $c_{i}$ ( $i=1,2,3,4)$ estimated by means of regression analysis are given in Table 2.

Table 1 gives the indication of an absolute magnitude gradient with respect to the colours for $M_{V}, M_{g}$ and $M_{J}$, whereas it is almost zero for $M_{K_{s}}$. We plotted the absolute magnitudes estimated for the bins of different colours in Table 1 versus the corresponding colour with (absolutely) larger coefficient, i.e. $(B-V)_{o},(J-H)_{o}$, and $(g-r)_{o}$ to treat the problem schematically. Fig. 8 confirms the result obtained via Table 1 , i.e. there is a linear variation of $M_{V}, M_{g}$ and $M_{J}$, whereas the locus of $M_{K_{s}}$ is a horizontal line.

We evaluated the differences between the absolute magnitudes estimated using the corresponding equation in (8) as the combination of apparent magnitudes and Hipparcos parallaxes of the stars, and plotted them in Fig. 9. The original values of the absolute magnitudes are those evaluated by the combination of apparent magnitudes and Hipparcos parallaxes of the stars. The standard deviations for the optical and NIR regions are $\sigma=0.23$ and $\sigma=0.28 \mathrm{mag}$, respectively. The dotted lines in the figure correspond to the residuals of $\pm 1 \sigma$.

\section{Test of Absolute Magnitudes of RC Stars}

We tested the calibrations of $M_{V}$ and $M_{K_{s}}$ absolute magnitudes on $101 \mathrm{RC}$ stars in the field SA 141. We estimated the Galactic model parameters with CCD $U B V R I$ photometric data for these RC stars and compared them with the ones appeared in the literature. The procedure is given in the following.

\subsection{Identification of the RC stars in SA 141}

Siegel et al. (2009) determined the CCD UBVRI magnitudes of 1299 stars in 1.2 square-degrees in a field in the direction of SA $141\left(l=246^{\circ} .33, b=-85^{\circ} .83\right)$. We used the $B$ and $V$ magnitudes of these stars, identified the RC stars in this field and estimated the Galactic model parameters by using the space densities evaluated for the RC sample as explained in the following. We de-reddened the $B$ and $V$ magnitudes by using the interstellar extinction maps of Schlegel et al. (1998)
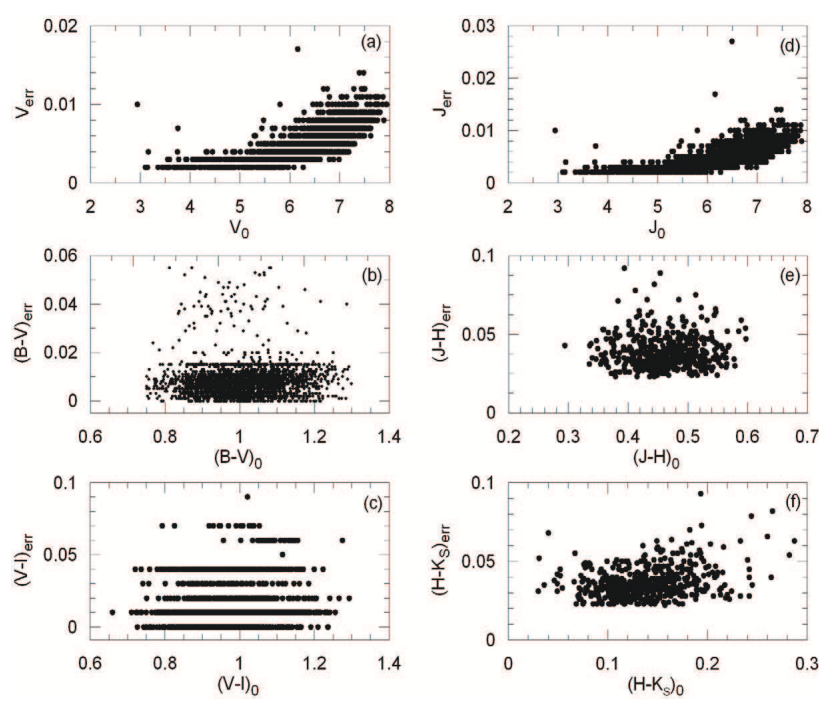

Fig. 7 Photometric errors for optical (a-c) and NIR (d-f) spectral regions.
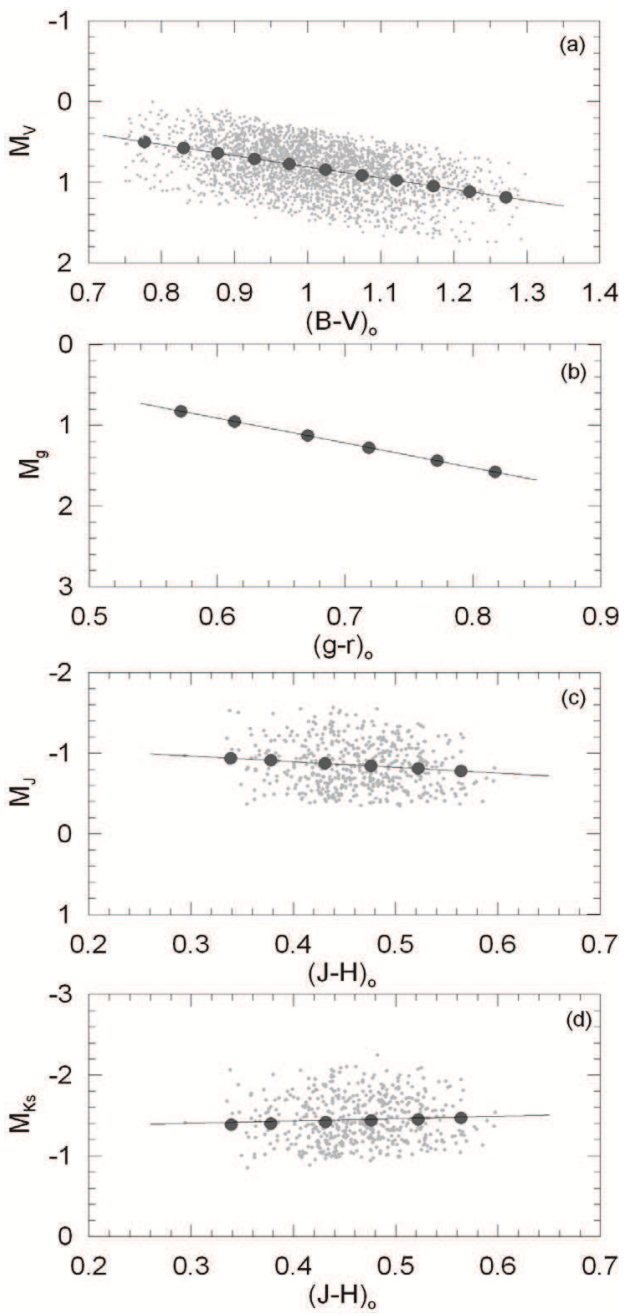

Fig. 8 Four absolute magnitude-colour diagrams for the RC stars: (a) $M_{V}-(B-V)_{0}$, (b) $M_{g}-(g-r)_{0}$, (c) $M_{J}-$ $(J-H)_{0}$, and $M_{K_{s}}-(J-H)_{0}$. 
Table 1 Distribution of colours and absolute magnitudes of the RC stars in 11 bins for the BVI photometry, and six bins for the $J H K_{s}$ and gri photometries.

\begin{tabular}{cccccccccc}
\hline \multicolumn{3}{c}{ Johnson-Cousins } & \multicolumn{3}{c}{ 2MASS } & \multicolumn{3}{c}{ SDSS } \\
$(B-V)_{o}$ & $(V-I)_{o}$ & $M_{V}$ & $(J-H)_{o}$ & $\left(H-K_{s}\right)_{o}$ & $M_{J}$ & $M_{K_{s}}$ & $(g-r)_{o}$ & $(r-i)_{o}$ & $M_{g}$ \\
\hline 0.777 & 0.777 & 0.502 & 0.339 & 0.146 & -0.937 & -1.386 & 0.571 & 0.179 & 0.825 \\
0.830 & 0.820 & 0.574 & 0.378 & 0.142 & -0.910 & -1.400 & 0.613 & 0.196 & 0.954 \\
0.877 & 0.863 & 0.639 & 0.431 & 0.137 & -0.873 & -1.419 & 0.671 & 0.219 & 1.131 \\
0.927 & 0.904 & 0.709 & 0.476 & 0.132 & -0.841 & -1.436 & 0.719 & 0.239 & 1.279 \\
0.975 & 0.945 & 0.775 & 0.522 & 0.130 & -0.809 & -1.452 & 0.772 & 0.261 & 1.440 \\
1.024 & 0.986 & 0.843 & 0.564 & 0.126 & -0.779 & -1.467 & 0.817 & 0.279 & 1.580 \\
1.074 & 1.028 & 0.913 & & & & & & & \\
1.122 & 1.096 & 0.979 & & & & & & & \\
1.172 & 1.144 & 1.048 & & & & & & & \\
1.222 & 1.201 & 1.118 & & & & & & & \\
1.271 & 1.227 & 1.186 & & & & & & & \\
\hline
\end{tabular}
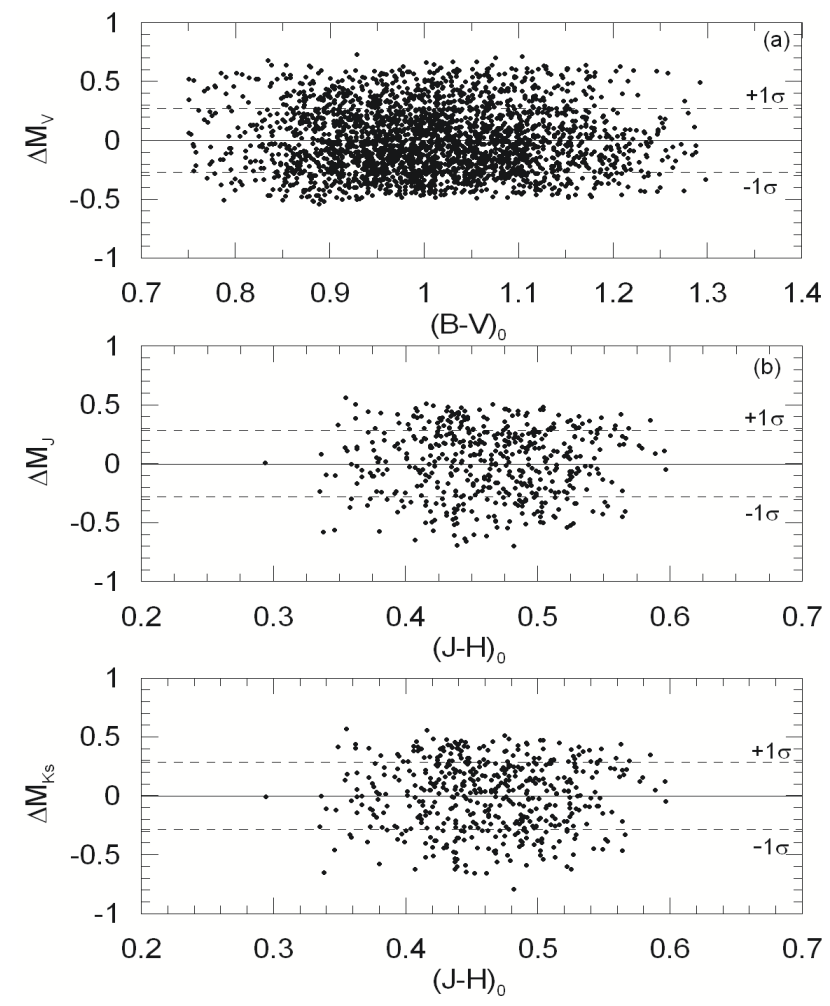

Fig. 9 Absolute magnitude residuals for $M_{V}, M_{J}$, and $M_{K_{s}}$. Dotted horizontal lines indicate $\pm 1 \sigma$ residuals. and the canonical procedure, as explained Section 2.2. The apparent $V$ magnitude of the stars lie in the interval of $12<V<22 \mathrm{mag}$ and their colour excesses are rather small, i.e. $0.010<E(B-V)<0.025 \mathrm{mag}$. We added 49 stars with $J H K_{s}$ magnitudes to this sample which were provided from 2MASS All Sky Catalog of point sources (Cutri et al. 2003) and which could not be observed with $U B V R I$ photometry due to the saturation of the CCDs. Thus, the number of stars in the field increased to 1348. The colour excess for the bright stars adopted as the mean of the colour excesses of 1299 stars, $E(B-V)=0.016$ mag.

The $V_{0}-(B-V)_{0}$ colour-magnitude diagram (CMD) of 1299 stars observed by Siegel et al. (2009) and the $K_{s_{o}}-\left(J-K_{s}\right)_{o}$ CMD of 859 stars provided by the 2MASS catalogue (Cutri et al. 2003) are given in Fig. 10. Bright stars which could not be observed with $U B V R I$ photometry are marked with the symbol $(+)$. The colour and magnitude errors for the $J H K_{s}$ photometry are larger than the ones for $U B V R I$ photometry (Fig. 11). However, 49 stars used in our study are bright ones with best quality labeled as "AAA". Hence, the large errors in question will not affect our results. The limiting magnitudes of completeness for $V_{0}$ and $K_{s_{0}}$ are 18.5 and $14.5 \mathrm{mag}$, respectively (Fig. 12).

The RC stars in Fig. 10 were identified in two steps. First, we applied the colour constraint of Puzeras et al.

Table 2 The numerical values of the coefficients $a_{i}, b_{i}$, and $c_{i}(i=1,2,3,4)$ in the Eq. (8).

\begin{tabular}{ccccc}
\hline$i \rightarrow$ & $(1)$ & $(2)$ & $(3)$ & $(4)$ \\
\hline & $M_{V}$ & $M_{g}$ & $M_{J}$ & $M_{K_{s}}$ \\
\hline$a_{i}$ & $1.398 \pm 0.010$ & $3.152 \pm 0.075$ & $0.706 \pm 0.017$ & $-0.337 \pm 0.013$ \\
$b_{i}$ & $-0.011 \pm 0.005$ & $-0.213 \pm 0.138$ & $0.039 \pm 0.019$ & $0.275 \pm 0.143$ \\
$c_{i}$ & $-0.577 \pm 0.001$ & $-0.937 \pm 0.075$ & $-1.182 \pm 0.034$ & $-1.312 \pm 0.025$ \\
$s$ & 0.0003 & 0.0009 & 0.0003 & 0.0002 \\
\hline
\end{tabular}



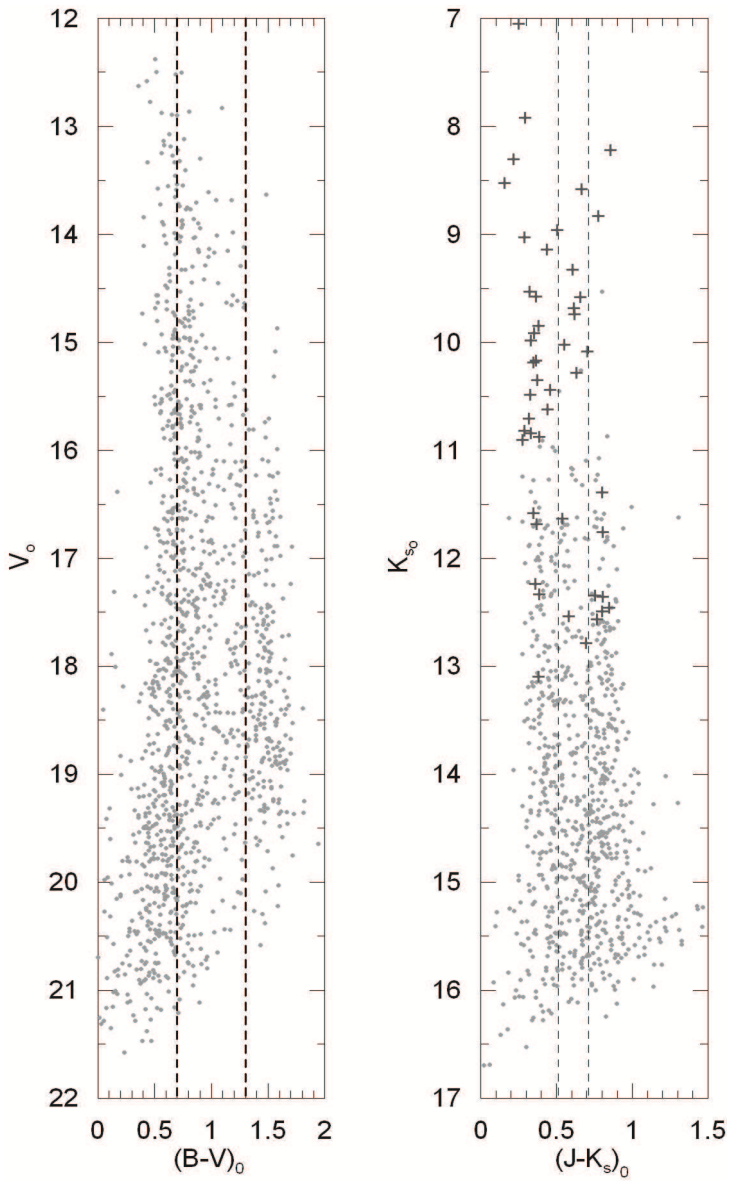

Fig. $10 V_{o}-(B-V)_{o}$ and $K_{s_{o}}-\left(J-K_{s}\right)_{o}$ apparent magnitude colour diagrams for the stars observed in the field SA 141. The symbol (+) denotes the bright stars which could not be observed with $B V I$ photometry due to saturation. The vertical dashed lines indicate the lower and upper limiting colours of the RC stars.
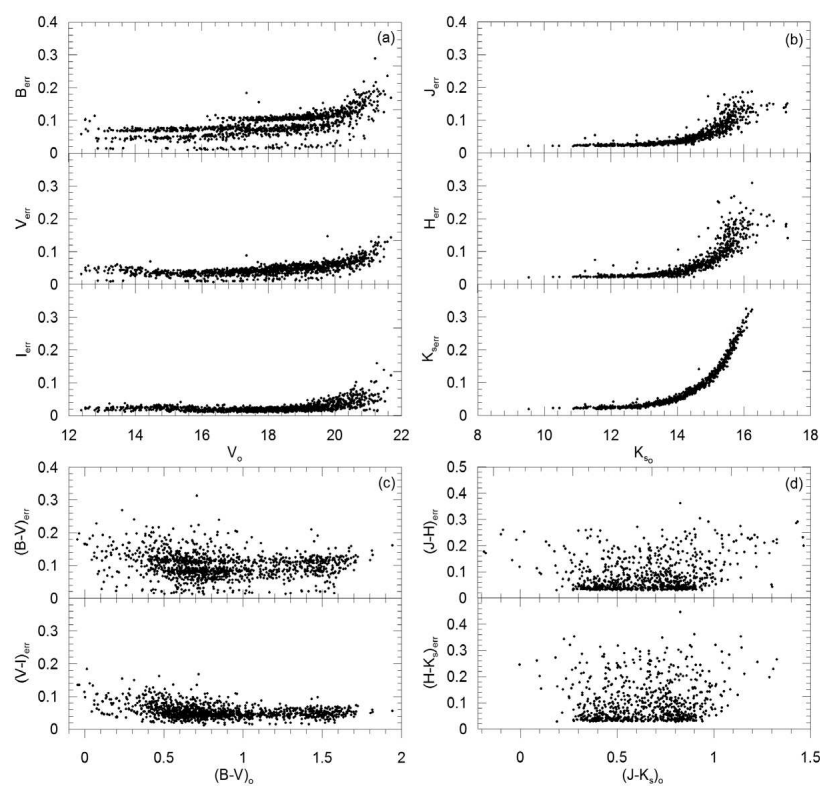

Fig. 11 Optical (a and c) and NIR (b and d) magnitude and colour errors for stars observed in SA 141 star field.
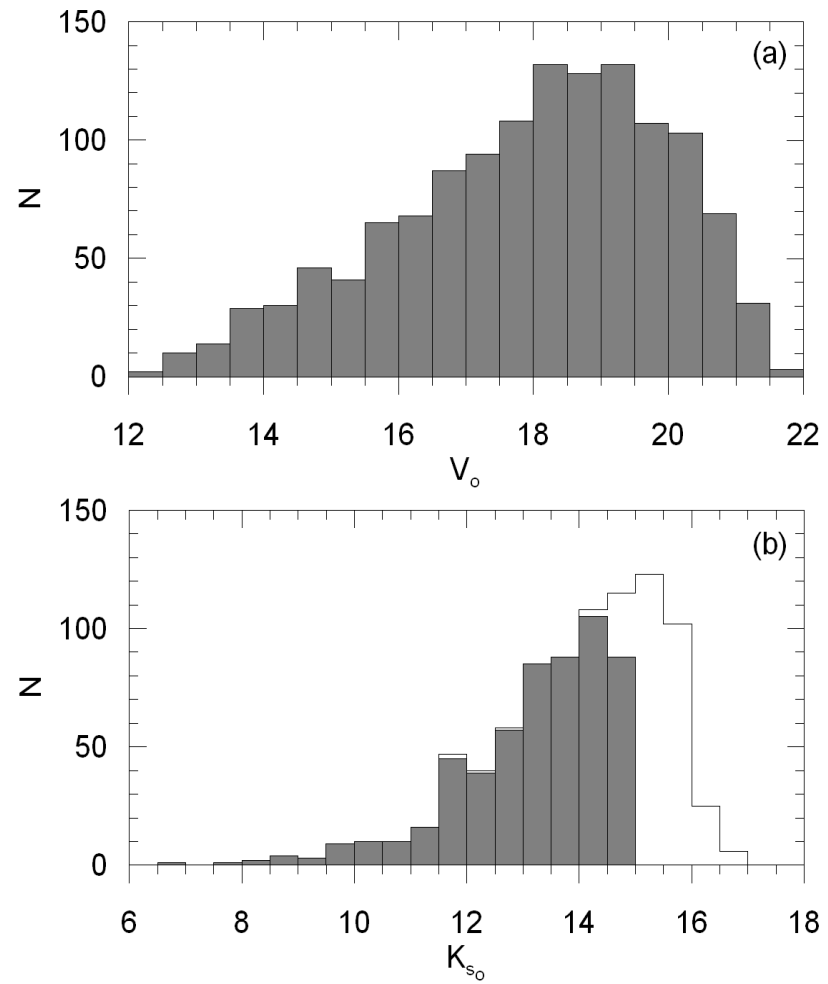

Fig. 12 Limiting magnitude of completeness for $V_{o}$ (a) and $K_{s_{o}}(\mathrm{~b})$ magnitudes. The histogram with colours white and black corresponds to all stars (859) observed with $J H K_{s}$ photometry, whereas the one with black colour is plotted only for the stars with best quality, labeled with AAA (563). 
$(2010)$, i.e. $0.7 \leq(B-V)_{0} \leq 1.3$ and $0.3 \leq(J-H)_{0} \leq$ $0.6 \mathrm{mag}$, and obtained a set of 249 dwarfs and giants. Then, we plotted them onto the $V_{0}-J_{0}$ two magnitude diagram (Fig. 13) and separated the giants (RC stars) from the dwarfs by the procedure of Bilir et al. (2006a). It turned out that 101 stars were classified as RC stars.

We evaluated the absolute magnitudes of $101 \mathrm{RC}$ stars and transformed them to distances by the Pogson formula,

$(m-M)_{0}=5 \log d-5$.

The $M_{V}$ absolute magnitudes for 90 stars with $B V I$ magnitudes were evaluated by the first equation in (8), whereas for $M_{K_{s}}$ absolute magnitudes for 11 stars with 2MASS magnitudes we used the last equation. The range of distance is $1<d \leq 28 \mathrm{kpc}$.

\subsection{Galactic Model Parameters Estimated with RC Stars}

\subsubsection{Density function}

We evaluated the logarithmic space densities $D^{*}=$ $\log D+10$ for $101 \mathrm{RC}$ stars in the distance range $1<r<24 \mathrm{kpc}$, using the distances mentioned above. Here, $D=N / \Delta V_{1,2} ; \Delta V_{1,2}=(\pi / 180)^{2}(A / 3)\left(r_{2}^{3}-r_{1}^{3}\right)$; $A$ denotes the size of the field (1.2 square-degrees); $r_{1}$ and $r_{2}$ are the lower and upper limiting distances of the volume $\Delta V_{1,2} ; N$ is the number of stars per unit absolute magnitude; $r^{*}=\left[\left(r_{1}^{3}+r_{2}^{3}\right) / 2\right]^{1 / 3}$ is the centroid distance of the volume $\Delta V_{1,2}$; and $z^{*}=r^{*} \sin b, b$ being the Galactic latitude of the field centre. The results are given in Table 3.

\subsubsection{Density laws}

We adopted the density laws of Basel group Buser et al. 1998, 1999). Disc structures are usually parameterized in the cylindrical coordinates using radial and vertical exponentials:

$D_{i}(x, z)=n_{i} e^{-z / H_{i}} e^{-\left(x-R_{o}\right) / h_{i}}$,

Table 3 Space density function for $101 \mathrm{RC}$ stars in the direction to the field SA 141, calculated by the distances evaluated via the absolute magnitudes based on the procedure in this study.

\begin{tabular}{cccccc}
\hline $\begin{array}{c}r_{1}-r_{2} \\
(\mathrm{kpc})\end{array}$ & $\begin{array}{c}\Delta V_{1,2} \\
\left(\mathrm{pc}^{3}\right)\end{array}$ & $\begin{array}{c}r^{*} \\
(\mathrm{kpc})\end{array}$ & $\begin{array}{c}z^{*} \\
(\mathrm{kpc})\end{array}$ & $N$ & $D^{*}$ \\
\hline $1-3$ & $3.17 \mathrm{E}(6)$ & 2.41 & 2.40 & 9 & 4.45 \\
$3-5$ & $1.19 \mathrm{E}(7)$ & 4.24 & 4.22 & 12 & 3.96 \\
$5-8$ & $4.72 \mathrm{E}(7)$ & 6.83 & 6.81 & 14 & 3.56 \\
$8-12$ & $1.48 \mathrm{E}(8)$ & 10.38 & 10.36 & 20 & 3.17 \\
$12-16$ & $2.89 \mathrm{E}(8)$ & 14.28 & 14.24 & 17 & 2.72 \\
$16-20$ & $4.76 \mathrm{E}(8)$ & 18.22 & 18.17 & 19 & 2.53 \\
$20-24$ & $7.10 \mathrm{E}(8)$ & 22.18 & 22.12 & 8 & 2.05 \\
$>24$ & - & - & - & 2 & - \\
\hline
\end{tabular}

where $z=r \sin b$ is the distance from Galactic plane, $x$ is the planar distance from the Galactic center, $R_{0}$ is the solar distance to the Galactic center $(8 \mathrm{kpc}$; Reid 1993), $H_{i}$ and $h_{i}$ are the scaleheight and scalelength, respectively, and $n_{i}$ is the normalized local density. The suffix $i$ takes the values 1 and 2, for the thin and thick discs.

The density law for spheroid component is parameterized in different forms. The most common is the de Vaucouleurs (1948) spheroid used to describe the surface brightness profile of elliptical galaxies.

$D_{s}(R)=n_{s} \exp \left[10.093\left(1-\left(R / R_{o}\right)^{1 / 4}\right)\right] /\left(R / R_{o}\right)^{7 / 8}$.

Here, $n_{s}$ is the normalized density at the solar radius, $R$ is the (uncorrected) galactocentric distance in spherical coordinates. $R$ has to be corrected for the axial ratio $\kappa=c / a$,

$R=\left[x^{2}+(z / \kappa)^{2}\right]^{1 / 2}$,

where,

$x=\left[R_{o}^{2}+r^{2} \cos ^{2} b-2 R_{0} r \cos b \cos l\right]^{1 / 2}$,

with $l$ and $b$ being the Galactic longitude and latitude, respectively.

\subsubsection{Galactic model parameters}

We estimated the Galactic model parameters by fitting the density functions in Table 3 derived from the observations (combined from the three population components) to a corresponding combination of the adopted population-specific analytical density laws (Fig. 14). The distance to a star in the line of sight $(r)$, in our sample, is rather close to its distance from the Galactic plane $(z)$, due to the position of the field SA 141, i.e. $b=-85^{\circ} .83$. Hence, the space densities and the density laws are given as a function of $z$, instead of $r$. We extrapolated the logarithmic density from the nearest point, $z^{*}=2.4 \mathrm{kpc}$, to the local space density for giants $\left(D^{*}=6.64\right)$ in the literature (Gliese 1969) and used the classical $\chi_{\text {min }}^{2}$ statistic to estimate Galactic model parameters, which is the most commonly used

Table 4 Galactic model parameters estimated by the space density function in Table 3.

\begin{tabular}{ccccccc}
\hline Thin Disc & \multicolumn{3}{c}{ Thick Disc } & \multicolumn{3}{c}{ Halo } \\
$n^{*}$ & $n^{*}$ & $\%$ & $H(\mathrm{pc})$ & $n^{*}$ & $\%$ & $\kappa$ \\
\hline 6.61 & $5.40_{-0.07}^{+0.05}$ & $5.80_{-0.9}^{+0.7}$ & $975_{-48}^{+48}$ & $4.02_{-0.07}^{+0.07}$ & $0.2_{-0.1}^{+0.1}$ & $0.89_{-0.08}^{+0.11}$ \\
\hline
\end{tabular}




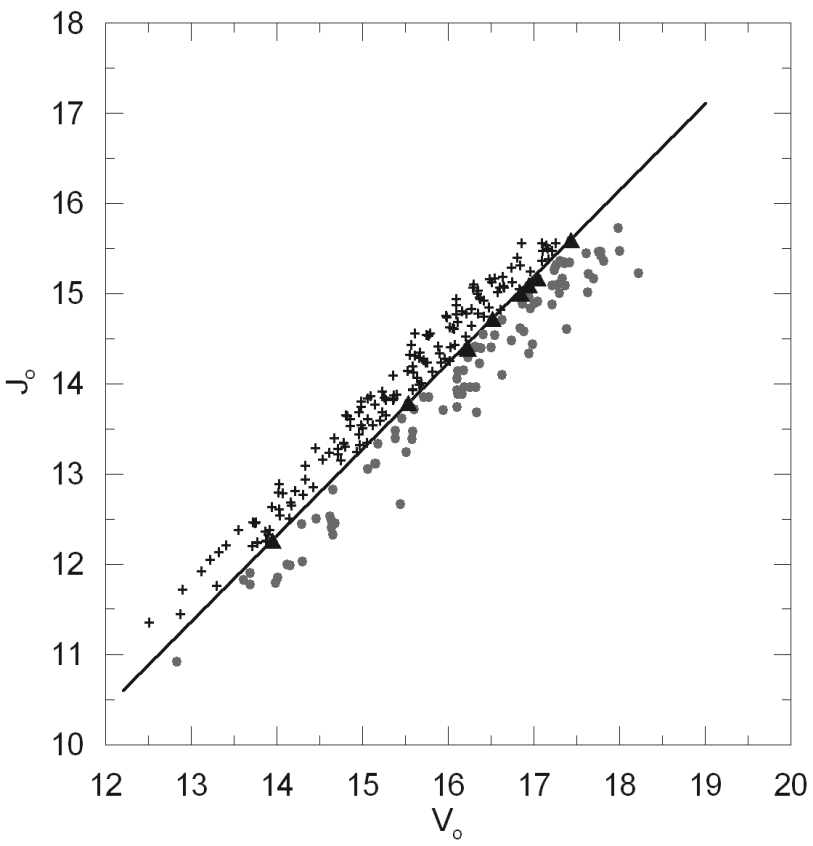

Fig. 13 Two magnitude diagram used for separation of dwarfs and giants (RC stars). Dwarf (plus), giant (circle), unclassified (triangle) due to their position on the diagram plus their errors. The solid line denotes the border of dwarfs and giants, adopted from Bilir et al. (2006a).

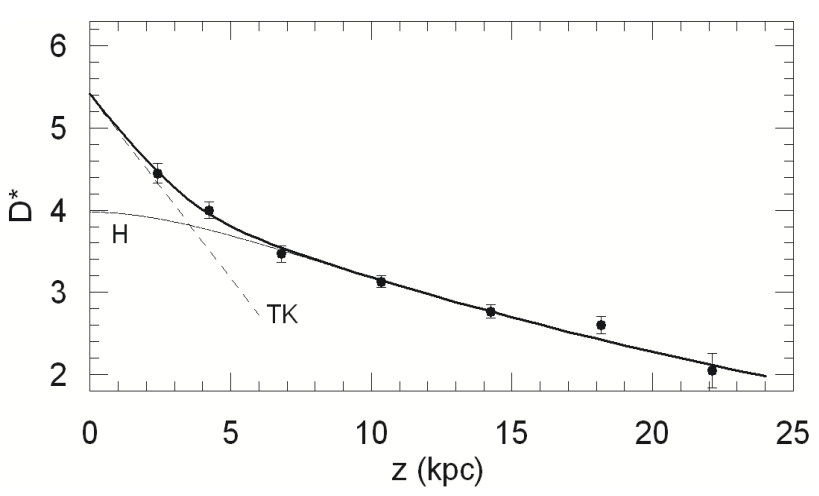

Fig. 14 Logarithmic space densities. The symbols TK and $\mathrm{H}$ denote the thick disc and halo components of the Galaxy, respectively. The curve indicates their combination. method in recent studies Du et al. 2006; Jurić et al. 2008; Bilir et al. 2008). The results are given in Table 4 . There is a good agreement between the Galactic model parameters estimated in this study and the ones appeared in the literature (cf. Karaali et al. 2004; Cabrera-Lavers et al. 2005; Bilir et al. 2006b.c).

\section{Discussion}

The RC stars are the most numerous population, within the red giants, and they are easy to detect at larger distances from the Sun. Hence, they become a powerful tool to trace the different Galactic components in the Milky Way. A lot of work has been done on this topic in recent years, but even more is still to come with the advent of the recent deeper databases in the NIR as those of UKIRT Infrared Deep Sky Survey (UKIDSS; Lawrence et al. 2007), or VISTA Variables in the Via Lactea (VVV) public survey (Minniti et al. 2010), or even with high quality data at larger wavelengths as the ones that the more recent Wide Field Survey Explorer (WISE; Wright et al. 2010) provides.

In recent years much work has been devoted to studying the suitability of RC stars for application as a distance indicator. The absolute magnitude of these stars in the $\mathrm{K}$ band is $M_{K_{s}}=-1.613 \mathrm{mag}$ with negligible dependence on metallicity (Alves 2000; Laney et al. 2012). Whereas, their absolute magnitudes in the optical range lie from $M_{V}=+0.7 \mathrm{mag}$ for those of spectral type G8 III to $M_{V}=+1$ mag for type K2 III (Keenan \& Barnbaum 1999).

In this work, we calibrated the $M_{V}, M_{g}, M_{J}$, and $M_{K_{s}}$ absolute magnitudes of RC stars in terms of colours. We found that the absolute magnitudes $M_{V}$ and $M_{g}$ are depend strongly on colour, whereas the $M_{J}$ and $M_{K_{s}}$ are weak. The calibrations of $M_{V}$ and $M_{K_{s}}$ absolute magnitudes are tested on $101 \mathrm{RC}$ stars in the field SA 141. The Galactic model parameters estimated with this sample are in good agreement with the ones appeared in the literature. The data of the RC stars are taken from the Hipparcos catalogue and only stars with relative parallax errors $\sigma_{\pi} / \pi \leq 0.1$ are considered to obtain reliable absolute magnitudes and distances. The range of the metallicity of stars in the Hipparcos catalogue is narrow. Hence, we did not consider the metallicity effect on the calibration of absolute magnitude in terms of colour.

\section{Acknowledgments}

This work has been supported by the Scientific and Technological Research Council (TÜBİTAK) $210 \mathrm{~T} 114$. 
We are grateful to Dr. Siegel for providing us the $U B V R I$ photometric data for the field SA 141 and Dr.

$T$. Güver for reading and correcting for grammatic and linguistic aspect of the manuscript.

This research has made use of the NASA/IPAC Infrared Science Archive and Extragalactic Database (NED) which are operated by the Jet Propulsion Laboratory, California Institute of Technology, under contract with the National Aeronautics and Space Administration.

This publication makes use of data products from the Two Micron All Sky Survey, which is a joint project of the University of Massachusetts and the Infrared Processing and Analysis Center/California Institute of Technology, funded by the National Aeronautics and Space Administration and the National Science Foundation.

This research has made use of the SIMBAD, and NASA's Astrophysics Data System Bibliographic Services. 


\section{References}

Alves, D.R.: 2000, ApJ, 539, 732

Bahcall, J.N., Soneira, R.M.: 1980, ApJS, 44, 73

Bilir, S., Karaali, S., Güver, T., Karataş, Y., Ak, S.: 2006a, AN, 327, 72

Bilir, S., Karaali, S., Gilmore, G.: 2006b, MNRAS, 366 , 1295

Bilir, S., Karaali, S., Ak, S., Yaz, E., Hamzaoğlu, E.: 2006c, NewA, 12, 234

Bilir, S., Cabrera-Lavers, A., Karaali, S., Ak, S., Yaz, E., López-Corredoira, M.: 2008, PASA, 25, 69

Bilir, S., Karaali, S., Ak, S., Önal, Ö., Dağtekin, N. D., Yontan, T., Gilmore, G., Seabroke, G. M.: 2012, MNRAS, 421, 3362

Buser, R., Rong, J., Karaali, S.: 1998, A\&A, 331, 934

Buser, R., Rong, J., Karaali, S.: 1999, A\&A, 348, 98

Cabrera-Lavers, A., Garzón, F., Hammersley, P.L.: 2005 , A\&A, 433, 173

Cabrera-Lavers, A., Hammersley, P.L., González-Fernández, C., López-Corredoira, M., Garzón, F., Mahoney, T.J.: 2007a, A\&A, 465, 825

Cabrera-Lavers, A., Bilir, S., Ak, S., Yaz, E., LópezCorredoira, M.: 2007b, A\&A, 464, 565

Cabrera-Lavers, A., González-Fernández, C.,Garzón, F., Hammersley, P. L., López-Corredoira, M.: 2008, A\&A, 491, 781

Cannon, R.D.: 1970, MNRAS, 150, 111

Cutri, R.M., Skrutskie, M.F., Van Dyk, S., et al.: 2003, The IRSA 2MASS All-Sky Catalog of Point Sources, NASA/IPAC Infrared Science Archive http://irsa.ipac.caltech.edu/applications/Gator

de Vaucouleurs, G.: 1948, Ann. d'Astrophys., 11, 247

Du, C., Ma, J., Wu, Z., Zhou, X.: 2006, MNRAS, 372, 1304

Fan, X.: 1999, AJ, 117, 2528

Fiorucci, M., Munari, U.: 2003, A\&A, 401, 781

Girardi, L., Bressan, A., Bertelli, G., Chiosi, C.: 2000, A\&AS, 141, 371

Girardi, L., Salaris, M.: 2001, MNRAS, 323, 109

Gliese, W.: 1969, Veröff. Astron. Recheninst. Heidelberg, No. 22

Groenewegen, M.A.T.: 2008, A\&A, 488, 25

Grocholski, A.J., Sarajedini, A.: 2002, AJ, 123, 1603

Jurić M.: et al, 2008, ApJ, 673, 864

Karaali, S., Bilir, S., Hamzaoğlu, E.: 2004, MNRAS, 355, 307

Keenan, P.C., Barnbaum, C.: 1999, ApJ, 518, 859

Kubiak, M., McWilliam, A., Udalski, A., Gorski, K.: 2002, AcA, 52, 159

Laney, C. D., Joner, M. D., Pietrzyński, G.: 2012, MNRAS, 419, 1637

Lawrence, A., et al.: 2007, MNRAS, 379, 1599

López-Corredoira, M., Cabrera-Lavers, A., Garzón, F., Hammersley, P.L.: 2002, A\&A, 394, 883

López-Corredoira, M., Cabrera-Lavers, A., Gerhard, O., Garzón, F.: 2004, A\&A, 421, 953

Lutz, T.E., Kelker, D.H.: 1973, PASP, 85, 573

Marigo, P., Girardi, L., Bressan A., Groenewegen, M.A.T., Silva, L., Granato, G.L.: 2008, A\&A, 482, 883

Marshall, D.J., Robin, A.C., Reylé, C., Schultheis, M., Picaud, S.: 2006, A\&A, 453, 635
Minniti, D., Lucas, P. W., Emerson, J.P., et al.: 2010, NewA, 15, 433

Páczyński, B., Stanek, K.Z.: 1998, ApJ, 494, L219

Pietrzyński, G., Gieren, W., Udalski, A.: 2003, AJ, 125, 2494

Pietrzyński, G., Górski, M., Gieren, W., Laney, D., Udalski, A., Ciechanowska, A.: 2010, AJ, 140, 1038

Puzeras, E., Tautvaišienè, G., Cohen, J.G., Gray, D. F., Adelman, S. J., Ilyin, I., Chorniy, Y.: 2010, MNRAS, 408, 1225

Reid, M.J.: 1993, ARA\&A, 31, 345

Salaris, M., Girardi, L.: 2002, MNRAS, 337, 332

Sarajedini, A.: 1999, AJ, 118, 2321

Schlegel, D.J., Finkbeiner, D.P., Davis, M.: 1998, ApJ, 500, 525

Skrutskie, M.F., et al.: 2006, AJ, 131, 1163

Siegel, M.H., Karataş, Y., Reid, N.: 2009, MNRAS, 395, 1569

Smith, H.Jr.: 1987, A\&A 171, 336

Stanek, K.Z., Garnavich, P.M.: 1998, ApJ, 503, L131

Trumpler, R. J., Weaver, H.F.: 1953, Statistical Astronomy, Dover Books on Astronomy and Space Topics, New York: Dover Publications

Udalski, A.: 2000, ApJ, 531L, 25

van Helshoecht, V., Groenewegen, M.A.T.: 2007, A\&A, 463, 559

van Leeuwen, F.: 2007, A\&A, 474, 653

Wright, E. L., et al.: 2010, AJ, 140, 1868

Yaz, E., Bilir, S., Karaali, S., Ak, S., Coşkunoğlu, B., Cabrera-Lavers, A.: 2010, AN, 331, 807

York, D.G., et al.: 2000, AJ, 120, 1579

Zhao,G., Qiu, H. M., Mao, S.: 2001, ApJ, 551L, 85

This manuscript was prepared with the AAS IATEX macros v5.2. 